\title{
Hurricane María drives increased indoor proliferation of filamentous fungi in San Juan, Puerto Rico: a two-year culture- based approach
}

\author{
Lorraine N Vélez-Torres ${ }^{1}$, Benjamín Bolaños ${ }^{\text {Corresp., }}{ }^{1}$, Filipa Godoy-Vitorino ${ }^{1}$, Félix E Rivera-Mariani ${ }^{2}$, Juan P \\ Maestre $^{3}$, Kerry Kinney ${ }^{3}$, Humberto Cavallín ${ }^{4}$ \\ ${ }^{1}$ Department of Microbiology \& Medical Zoology, Universidad de Puerto Rico, Recinto Ciencias Médicas, San Juan, PR, USA \\ 2 College of Biomedical Sciences, Larkin University, Miami, Florida, USA \\ 3 Department of Civil, Architectural, and Environmental Engineering, The University of Texas at Austin, Austin, Texas, USA \\ 4 School of Architecture, Universidad de Puerto Rico, Recinto de Rio Piedras, San Juan, PR, USA \\ Corresponding Author: Benjamín Bolaños \\ Email address: benjamin.bolanos@Upr.edu
}

The extensive flooding caused by Hurricane María in Puerto Rico (PR) created favorable conditions for indoor growth of filamentous fungi. These conditions represent a public health concern as contamination by environmental fungi is associated with a higher prevalence of inflammatory respiratory conditions. This work compares culturable fungal spore communities present in homes that sustained water damage after Hurricane María to those present in dry, non-flooded homes. We collected air samples from 50 houses in a neighborhood in San Juan, PR, 12 and 22 months after Hurricane María. Self-reported data was used to classify the homes as flooded, water-damage or dry non-flooded. Fungi abundances, composition and diversity were analyzed using tools originally develop for the data generated by molecular biology approaches (MicrobiomeAnalyst and METAGENassist platforms). Our results showed no significant differences in indoor fungal concentrations $\left(\mathrm{CFU} / \mathrm{m}^{3}\right)$ during the first sampling period in both culture media studied (MEA and G25N). During the second sampling period fungal levels were 2.6 times higher in flooded homes (Median $=408$ ) when compared to dry homes (Median $=233$ ), respectively ( $p$-value $<$ 0.005). Fungal profiles showed enrichment of Aspergillus species inside flooded homes compared to outdoor samples during the first sampling period (FDR-adjusted $p$-value $=$ 0.05). In contrast, during the second sampling period, the indoor fungal composition consisted primarily of non-sporulated fungi, most likely basidiospores, which are characteristic of the outdoor air in PR. Together, this data highlights that homes that suffered water damage not only have higher indoor proliferation of filamentous fungi, but their indoor fungal populations change over time following the hurricane. Ultimately, after nearly two years, the indoor and outdoor fungal communities converged in this sample of 
naturally ventilated homes. 
1 Hurricane María drives increased indoor proliferation of filamentous fungi in San Juan,

2 Puerto Rico: a two-year culture-based approach

3

4

5 Lorraine N. Vélez-Torres ${ }^{1}$, Benjamín Bolaños-Rosero ${ }^{1}$, Filipa Godoy-Vitorino ${ }^{1}$, Félix E. Rivera6 Mariani $^{2}$, Juan. P. Maestre ${ }^{3}$, Kerry Kinney ${ }^{3}$, Humberto Cavallín ${ }^{4}$

${ }^{1}$ Department of Microbiology and Medical Zoology, University of Puerto Rico-Medical Sciences Campus, San Juan, PR, USA

${ }^{2}$ College of Biomedical Sciences, Larkin University at Miami, Florida, USA

${ }^{3}$ Department of Civil, Architectural, and Environmental Engineering, The University of Texas at Austin, Texas, USA

${ }^{4}$ School of Architecture, University of Puerto Rico-Río Piedras Campus, San Juan, PR, USA

Corresponding Author

Benjamín Bolaños-Rosero

Microbiology Department, PO Box 365067, San Juan, PR, 00936, USA

Email address: benjamin.bolanos@upr.edu

(1)


42 The-extensive flooding caused by Hurricane María in Puerto Rico (PR) created favorable

43 anditions for indoor growth of filamentous fungi. These conditions represent a public health

44 concern as contamination by environmental fungi is associated with a higher prevalence of

45 inflammatory respiratory condition: his work compares culturable fungal spore communities

46 present in homes that sustained water damage after Hurricane María to those present in dry, non-

47 flooded homes. We collected air samples from 50 houses in a neighborhood in San Juan, PR, 12

48 and 22 months after Hurricane María. Self-reported data was used to classify the homes as

49 flooded, water-damage or dry non-flooded. Fungi abundances, composition and diversity were

50 analyzed using tools originally develop for the data generated by molecular biology approaches

51 (MicrobiomeAnalyst and METAGENassist platforms). Our results showed no significant

52 differences in indoor fungal concentrations $\left(\mathrm{CFU} / \mathrm{m}^{3}\right)$ during the first sampling period in both

53 culture media studied (MEA and G25N). During the second sampling period fungal levels were

542.6 times higher in flooded homes (Median = 408) when compared to dry homes (Median = 233),

55 respectively-(p-value $<0.005)$. Fungal profiles showed enrichment of Aspergillus species inside

56 flooded homes compared to outdoor samples during the first sampling period (FDR-adjusted p-

57 value $=0.05$ ). In contrast, during the second sampling period, the indoor fungal composition

58 consisted primarily of non-sporulated fungi, most likely basidiospores, which are characteristic of

59 the outdoor air in PR. Together, this data highlights that homes that suffered water damage not

60 only have higher indoor proliferation of filamentous fungi, but their indoor fungal populations

61 change over time following the hurricane. Ultimately, after nearly two years, the-indoor and

62 outdoor fungal communities converged in this sample of naturally ventilated homes.

63

64

Keywords: Hurricane María, mold spores, fungi, mycobiome, aeroallergens, indoor air, indoor environment, Aspergillus, built environment, flooded homes, water-damage

\section{INTRODUCTION}

Fungi are ubiquitous eukaryotic organisms of environmental and medical importance as 
84 in the air with fungal spore sizes ranging from approximately $0.65 \mu \mathrm{m}$ to $>20 \mu \mathrm{m}$ (Portnoy, 85 Barnes \& Kennedy, 2008; Hamilos, 2010; Li et al., 2011; Kwon-Chung \& Sugui, 2013; Claub, 86 2015). Fungal spores and fragments less than $2.5 \mathrm{~m}$ can deposit in the alveoli and trigger asthma 87 in sensitized individuals (Joubert et al., 2020). In contrast, large fungal spores and clusters of 88 small fungal spores can deposit in the upper airway triggering allergic rhinitis (Joubert et al., 89 2020). Environmental fungal contamination, including high airborne fungal spore concentrations 90 in the air, are associated with inflammatory respiratory conditions such as asthma and allergic 91 rhinitis (Lewis et al., 2019). In addition, for people already suffering from asthma, exposure to 92 fungal spores simultaneously with other allergens (animal dander, dust mites, cockroaches) may 93 cause dangerous exacerbations of existing asthma (Gautier \& Charpin, 2017). Approximately, 94339 to 500 million people worldwide are affected by asthma and allergic rhinitis, respectively 95 (Kołodziejczyk \& Bozek, 2016; Global Asthma Network, 2018). In Puerto Rico (PR), asthma 96 prevalence in children and adults is higher than in the United States (2017: 15.5\% vs 7.5\% for 97 children and 12.2\% vs. 9.1\% for adults) (Ortiz-Rivera, 2018).

98 Due to its tropical location, frequent hurricanes and extreme rain events, PR has a high 99 concentration of fungal spores in the air. Very high levels observed in PR exceed 110,000 spores/ $100 \mathrm{~m}^{3}, 2.2$ times higher than those observed in the other National Allergy Bureau (NAB) of the 101 American Academy of Allergy Asthma and Immunology mold levels stations (AAAAI, 2021). 102 Outdoor fungal spore concentrations typically reach their maximum levels in PR during the rainy 103 months of September to November (Quintero, Rivera-Mariani \& Bolaños-Rosero, 2010). The 104 fungi found in outdoor ambient air are typically split between Basidiomycetes (60\%) and 105 Ascomycetes (40\%) (Rivera-Mariani et al.; AAAAI, 2021). Pleurotus ostreatus, Chlorophyllum 106 molybdites and Ganoderma applanatum are some of the basidiomycetes common in the air of PR 107 (Rivera-Mariani et al., 2011). Ascomycetes include filamentous Aspergillus species (A. fumigatus, A terreus, A. niger, A. flavus) which are the most frequent causative agents of human aspergillosis, lung inflammation and among the most frequent triggers of asthma and allergic rhinitis (Portnoy, Barnes \& Kennedy, 2008; Hamilos, 2010; Kwon-Chung \& Sugui, 2013). Indeed, a recent study in PR found that increases in outdoor fungal spore concentrations were associated with increases in asthma-related health claims, confirming that fungal spores are triggers of asthma (Lewis et al., 2019). Most Puerto Rican homes have natural air ventilation;

114 therefore, spores and pollen present outdoors can easily penetrate indoors through open windows 115 and doors. Even though the 2017 outdoor fungal spore season was lower than expected as a result 116 of Hurricane María, the fungal spore season for 2018 was the highest ever recorded in the 16-year 117 history of data collection at the San Juan station most likely due to the accumulation of organic 118 debris in the aftermath of Hurricane María (AAAAI, 2021; Bolaños-Rosero, 2021).

The-essential conditions for indoor growth of filamentous fungi include high moisture and 120 humidity, warm temperature, poor ventilation or air circulation, and the presence of organic 121 source materials (Institute of Medicine, 2004; WHO, 2009). Taking these factors into 122 consideration, the extensive flooding caused by Hurricane María in PR had the potential to 123 provide a favorable environment for fungal proliferation indoors in homes with water damage. 124 Several studies have evaluated the effects of hurricanes on airborne fungi in the United States, 125 with the most common fungi isolated being Aspergillus, Penicillium and Cladosporium (CDC, 
126 2006; Chew et al., 2006; Rao et al., 2007; Schwab et al., 2007; Rabito et al., 2008; Saporta \& 127 Hurst, 2017; Gargano et al., 2018; Chow et al., 2019; Kontoyiannis et al., 2019). Although PR

128 has been affected by more than 15 hurricanes and tropical storms since the 1980's, the effect of

129 these natural disasters on the concentration and composition of indoor airborne fungi have not yet

130 been studied. Here, we provide a critical and quantitative analysis of the airborne fungal

131 concentrations in the months following Hurricane María in PR. Our hypothesis is that homes

132 which sustained flooding or water damage will have higher fungal diversity and abundance of

133 fungi related with damp indoor environments. In addition, since the sampled homes are

134 predominately naturally ventilated, we hypothesize that fungal taxa in the indoor environment of

135 dry non-flooded homes may reflect the taxa in the outdoor environment. We believe this study

136 will provide the basis for understanding the effect of fungal growth and species composition as a

137 result of a flooding event in PR. The results from this study lay the foundation to design

138 mitigation strategies to minimize adverse fungal exposures after flooding events to better control

139 lung inflammation and asthma in PR and other tropical countries.

\section{MATERIALS AND METHODS}

Study Population. After obtaining Institutional Review Board (IRB) approval from the University of Puerto Rico - Río Piedras Campus (IRB protocol 1718-058), 50 residences from the area of Santurce (San Juan, PR) were selected to participate in this study (Fig. 1). Many homes located in this area sustained flooding during and after Hurricane María's passage through the island. Of the 50 homes, 10 were located in a non-flooded area and 40 were located in a flooded area according to a reference map generated by the Federal Emergency Management Agency (FEMA). In addition to considering this classification, participants were surveyed and asked to describe in more detail the level of water damage. This reported data allowed the classification of each household in greater detail: 13 houses were dry non-flooded, 24 houses had water-damage (received water from above, roof and/or windows), and 13 houses were flooded (received water from below, through doors and crevices). We acknowledge that the sample size for the different categories was small, but we were working with recruitment under very difficult circumstances. We thus have two types of classifications for each home: (1) FEMA based: whether the homes were located in a flooded or non-flooded area according to FEMA (dry area or flooded area) and (2) Self-reported damage reported by study participants (dry, water-damage or flooded). After obtaining written informed consent, participants were asked to complete questionnaires in order to gather information on the demographics, health of home occupants (respiratory and psychological), home characteristics (ventilation, number of windows, construction materials) and the physical impact of Hurricane María on the home (water-damage, remediation activities, and the presence of visible mold). For the purpose of this study, we focus on evaluating the relationship between the self-reported degree of water damage and viable fungal communities

166 Air Sampling \& Fungal Culturing. We collected indoor and outdoor samples during two different 167 sampling periods: August - September 2018 and June-July 2019, 12 and 22 months, respectively, 
168 after Hurricane María. For the air sample collection, we used the MicroBio MB2 Bioaerosol

169 Sampler (Cantium Scientific, Dartford, UK). This volumetric instrument collects airborne

170 bioaerosol contaminants by aspirating air through a removable stainless-steel head (cover) which

171 contains 220, $1 \mathrm{~mm}$ holes. This design creates a laminar airflow that impels the microorganisms,

172 in this case the fungal spores, onto the surface of the culture media. A pilot study using different

173 sampling volumes allowed us to set the optimal total air sampling volume at 60 liters (flow rate

174 of 60L/35 seconds) which allowed us to count and identify to genus level the viable fungi without

175 overgrowth on the Petri plate. Prior to Hurricane María, the standard sampling volume used in a

176 previous study was 150 liters, but due to record-high outdoor fungal spores levels reported in

177 2018, the sampling volume was decreased to 60 liters (Bolaños-Rosero et al., 2013). At each

178 home, indoor samples were collected at a height of 1 meter from four indoor locations (living

179 room, kitchen, bedroom and bathroom) and an outdoor sample was collected in the front yard of

180 the residences. Before sample collection, interior fans were turned off and the number of open

181 and closed windows were counted. We used two types of culture media, Malt Extract Agar

182 (MEA) and 25\% Glycerol Nitrate Agar (G25N; $\mathrm{K}_{2} \mathrm{HPO}_{4} 0.375$ g, Czapek concentrate 3.75 ml,

183 yeast extract $1.75 \mathrm{~g}$, glycerol $125 \mathrm{ml}$, agar $6 \mathrm{~g}$, distilled water $375 \mathrm{ml}$ ). MEA is a nutritive

184 growth media used for the isolation of general fungi since it is suitable for most fungal growth

185 while the G25N is a selective growth media for xerophilic (dry tolerant) fungi, which grow at low

186 water activity or availability (Wu, Su \& Ho, 2000; De León et al., 2018). After sampling, the

187 Petri plates were incubated at $25 \pm 2^{\circ} \mathrm{C}$ for up to 2 weeks. Visible fungal colonies were counted

188 using a stereomicroscope as early as 24-48 hours after sampling with MEA, taking precautions to

189 avoid mixing of the fungal colonies such as keeping the inverted plates horizontally. This process

190 was repeated daily for 7 days in the case of MEA and for 14 days in the case of G25N. Culturable

191 fungi were reported in colony forming units per cubic meter $\left(\mathrm{CFU} / \mathrm{m}^{3}\right)$ using the MicroBio PC

192 Reporter software and data from the fungal colony count with positive-hole correction and the

193 total volume of air drawn through the MicroBio MB2 Bioaerosol sampler for each sample

194 collected. The-positive-hole correction accounts for the probability of more than one viable

195 particle could be entering through a sampling hole, merging with other microorganisms at the

196 same impaction site and producing a single colony(Macher, 1989). In addition to determining

197 culturable fungi at each participating home, we also measured relative humidity levels both

198 indoor and outdoor at each home. We also compiled precipitation data from the National Weather

199 Service Forecast Office (NWSFO) and outdoor fungal spore levels from the San Juan AAAAI

200 station for both sampling periods to characterize the ambient fungal background levels during the

201 sampling events (NWSFO, 2014; AAAAI, 2021).

202

203 Fungal Identification. After fungal colonies were counted,-we performed slide mounts to analyze

204 the colonies under the microscope and identify them to the genus level. First, we used the

205 stereomicroscope to evaluate how many possible distinct fungi we had in our sample. Prior to

206 preparing the slide mounts, we isolated the most predominant fungi present for further

207 experiments. Then, we used fungi tape and lactophenol cotton blue staining to prepare the slide

208 mounts. Following this, we observed the fungal slide mounts under the microscope and the fungi 
209 were identified to the genus level by evaluation of morphological characteristics such as spore

210 shape, size, color and arrangement (Samson et al., 2019).

211

212 Statistical Tests and Data Analyses. Data on fungal colonies concentrations and metadata

213 variables were analyzed in RStudio@ Version 1.2.1335 (R Foundation for Statistical Computing,

214 Vienna, Austria) (RStudio-Team, 2020). When analyzing the indoor data, we used the value of

215 the calculated median of the indoor measurements per home. To evaluate differences in indoor

216 relative humidity by degree of water damage (Dry, Water-Damage, Flooded), we performed the

217 non-parametric Kruskal Wallis test. We also used the non-parametric test Kruskal-Wallis to

218 analyze differences in fungal colonies concentrations as a function of different self-reported

219 degree of water damage (dry, water-damage, flooded). We considered a p-value less than 0.05

220 statistically significant. To evaluate the contribution of outdoor fungal levels to indoor fungal

221 levels, we performed a Spearman Correlation analysis and a Linear Regression analysis with

222 RStudio for graphs and Intellectus Statistics for interpretation (Intellectus Statistics; RStudio-

223 Team, 2020). For the purposes of performing alpha and beta diversity analyses and visualization

224 of the plots, we used MicrobiomeAnalyst and METAGENassist which are web-based platforms

225 used for the analysis of microbiota data (Arndt et al., 2012; Dhariwal et al., 2017). Biodiversity

226 census, either culture-based or culture-independent, require a consensus on diversity metrics.

227 That is, standardized methods for the measurement of biodiversity at different scales exist, such

228 as Chao1 observed species metrics or diversity indexes of Shannon or Simpson, initially used to

229 monitor biodiversity (Roswell, Dushoff \& Winfree, 2021). Here, we cataloged the grown molds

230 (culture-based approach) and used the species table of abundances to calculate alpha and beta

231 diversity estimates. For data filtering, processing and normalization, we used a low-count filter

232 based on 10\% prevalence and no data rarefaction was performed. Normalization was done row-

233 wise, log transformed on the total sum, in METAGENassist so that the data could follow a

234 Gaussian distribution and samples could be more comparable to each other. Statistical analyses

235 for alpha diversity, were done using compare_alpha_diversity.py in QIIME which uses a non-

236 parametric t-test with Monte Carlo permutations. For Beta-diversity analyses, PCoA, Jensen-

237 Shannon Divergence and permutational MANOVA (PERMANOVA) were used as ordination,

238 distance and statistical methods, respectively. Taxonomic bar plots were created using percentage

239 abundance and a taxa resolution of the top $n$ taxa, with $n=10$. To identify significant taxa

240 signatures at genus/species level between two different groups, a Linear Discriminant Analysis

241 (LDA) Effect Size (LEfSe) algorithm for biomarker discovery was used (Segata et al., 2011).

242 This method allowed us to identify statistically significant fungal features that differ between the

243 indoor and outdoor environments of homes classified by self-reported degree of water damage,

244 expressed as LDA values shown in boxplots and using either Original (uncorrected) or FDR

245 (False Discovery Rate)-adjusted p-value < 0.05). In addition, we compared the fungal profile

246 (alpha, beta, abundance and biomarker analysis) between dry and wet (water-damage + flooded)

247 homes.

248

249

\section{RESULTS}

250 
251 During the first sampling period, a total of 50 homes were sampled (Fig. 1). For the

252

253

254

255

256

257

258

259

260

261

262

263

264

265

266

267

268

269

270

271

272

273

274

275

276

277

278

279

280

281

282

283

284

285

286

287

288

289

290

291

292 second sampling period, a total of 35 homes completed the follow-up sampling event (70\% retention rate). During the first sampling period in 2018, 10 homes were in a non-flooded zone and 40 were in a previously flooded zone as per FEMA's classification scheme following Hurricane María (Table 1). For the second sampling period in 2019, 8 of 35 homes were in a dry FEMA zone and 27 in a previously flooded area according to FEMA. The self-reported data on flooding conditions provided by participants yielded a more detailed assessment of actual flooding and water damage conditions within the study homes. It is worth noting that the FEMA classification does not take into account details that can influence the extent of the flooding (e.g. some homes were on second floors and were not flooded even though they were located in an area categorized by FEMA as flooded). Of the 50 homes sampled in 2018, 13 houses were reported by household members to be dry, 24 had water-damage, and 13 had been subjected to flooding. Of the 35 homes sampled in 2019, 11 houses were reported by household members to be dry, 15 had water-damage, and 9 had been subjected to flooding. All the homes were normally found to be naturally ventilated via open doors and windows. Fans and/or an occasional air conditioner were also present in some homes but these were turned off during air sample collection.

No significant differences in median relative humidity levels inside dry, water-damage and flooded homes were observed during the first sampling period (Fig. 2A). In contrast, the indoor environment of flooded homes had significantly higher relative humidity levels during the second sampling period when compared to dry homes (p-value $=0.007)$ (Fig. 2B).

Since outdoor fungal spore concentrations are important in the context of tropical homes with natural ventilation, we reviewed these data for the sampling periods of our study. Outdoor levels of fungal spores, reported at the San Juan AAAAI station, were higher during the first sampling period (an average of 86,478 spores $/ \mathrm{m}^{3}$ and 103,762 spores $/ \mathrm{m}^{3}$ in August and September of 2018, respectively) when compared to the second sampling period (an average of 44,615 spores $/ \mathrm{m}^{3}$ and 38,632 spores $/ \mathrm{m}^{3}$ in June and July of 2019, respectively). Similar results were obtained for outdoor culturable fungi $\left(\mathrm{CFU} / \mathrm{m}^{3}\right)$ measured outside the study homes with 1.88- and 1.12-times higher levels during the first sampling period when compared to the second sampling period in G25N and MEA, respectively (data not shown, not statistically significant). As outdoor San Juan fungal spore concentrations follow a seasonal pattern related to seasonal precipitation, the precipitation levels during the sampling periods were also compared (Quintero, Rivera-Mariani \& Bolaños-Rosero, 2010). Precipitation levels were higher in August 2018 (first sampling period, total monthly precipitation of 6.29") and in July of 2019 (second sampling period, total monthly precipitation of 6.72") (NWSFO, 2014).

We evaluated the relationship between outdoor and indoor fungal concentration (CFU/ $\mathrm{m}^{3}$ ). For indoor levels we used the culturable measurements median of the indoor locations at each home. For the first sampling period, 12 months after Hurricane María, a significant positive correlation was observed between-outdoor and indoor fungal concentration $\left(\mathrm{CFU} / \mathrm{m}^{3}\right)(r=$ 
$2930.47, p<.001,95 \% \mathrm{CI}=[0.22,0.66])$. This correlation indicates that as outdoor fungal colonies

294 increase $e_{2}$ indoor fungal colonies also tend to increase. The results of the linear regression model

295 were significant, $F(1,48)=60.79, p<.001, R^{2}=0.56$, indicating that approximately $56 \%$ of the

296 variance in indoor fungal colonies is explainable by the outdoor levels (Fig. 3A). For the second

297 sampling period, 22 months after Hurricane María, a significant positive correlation was still

298 observed between outdoor and indoor (living room) fungal colonies $(r=0.71, p<.001,95 \% \mathrm{CI}=$

$299[0.49,0.84])$. The correlation coefficient between outdoor and indoor was 0.71 , indicating a large

300 effect size. The results of the linear regression model were also significant, $F(1,33)=$

$30111.40, p=.002, R^{2}=0.26$, with approximately $26 \%$ of the variance in indoor fungal colonies

302 explained by outdoor fungal colonies (Fig. 3B).

303

304

305

306

307

308

309

310

311

312

313

314

315

316

We evaluated indoor culturable fungi concentrations in the study homes as a function of self-reported degree of water damage. During the first sampling period in 2018, no significant differences in culturable fungi concentration were observed in both culture media evaluated (Fig. 4A and $4 \mathbf{B}$ ). For the second sampling period, indoor $\mathrm{CFU} / \mathrm{m}^{3}$ increased with degree of water damage for both culture media (Fig. 4A and 4B). Both flooded (Median = 758) and waterdamage homes (Median $=408$ ) had 2.6 and 1.4 times higher $\mathrm{CFU} / \mathrm{m}^{3}$ when compared to dry nonflooded homes (Median $=233$ ) in the G25N culture media (Fig. 4A), although only statistically significant between flooded and dry homes (p-value $<0.005$ ). Results from the MEA culture media (Fig. 4B) during the same period showed 2 and 1.6 times higher $\mathrm{CFU} / \mathrm{m}^{3}$ in flooded (Median $=716)$ and water-damage homes (Median $=583$ ) when compared to dry, non-flooded homes (Median $=358$ ), although only statistically significant between flooded and dry homes (pvalues $<0.01)$.

330

331

We also-analyzed the culture-based data using web-based platforms originally designed for visualizing molecular data. Beta-diversity analysis using PCoA showed statistical differences (p-values $<0.006$ ) between fungal communities as a function of self-reported degree of water damage during the first sampling period (Fig. 4C). Results from alpha-diversity analysis using the Chao1 richness estimator, showed no significant differences ( $p$-value $=0.38415)$ in richness by self-reported degree of water damage (Fig. 4D, Supplementary Table 1).

We then compared the relative abundance of fungal taxonomic features at the genus level by sampling location (indoor or outdoor) and self-reported degree of water damage during both sampling periods (first and second) (Fig. 5A and 5B). During the first sampling period, the fungal taxa present in the indoor environment of dry homes had similar relative abundance levels to those present in the outdoor environment and were dominated by non-sporulating fungi (Fig. 5A). The-water-damage homes were still dominated by non-sporulating fungi, but there was an increase of Aspergillus taxa in the indoor environment. Specifically, during the first sampling period, the indoor environment of flooded homes was enriched by Aspergillus species when compared to the outdoor samples. During the second sampling period, enrichment of Aspergillus taxa in flooded homes was not observed (Fig. 5B). Both indoor and outdoor environments were dominated by non-sporulating fungi regardless of the degree of water-damage in the homes sampled. Linear discriminant analysis (LDA) together with effect size measurement (LEfSe) 
335 method was used in order to identify statistically significant fungal features at the genus/species

336 level (Fig. 5C and 5D). Higher LDA scores represent increased abundance of fungal biomarkers

337 in the samples classified by sampling location (indoor versus outdoor) and self-reported degree of

338 water damage (dry, water-damage, flooded). During the first sampling period, enrichment of non-

339 sporulating fungi was identified in the outdoor samples of dry homes, whereas Aspergillus

340 tamari and Rhodotorula were significantly enriched in the outdoor samples of water-damage and

341 flooded homes, respectively (Fig. 5C). In contrast, Aspergillus was significantly enriched in the

342 indoor environment of flooded homes during the first sampling period. During the second period

343 no significant features were found.

Finally, we analyzed the abundance levels of fungal taxa individually indoors versus outdoors (Fig. 6). During the first sampling period, Aspergillus spp. and Penicillium spp. were more abundant in indoor samples, whereas non-sporulating fungi and Cladosporium spp. were more abundant outdoors (Fig. 6A). For the second sampling period, Trichoderma was more abundant indoors while Aspergillus, non-sporulating fungi and Eurotium amstelodami were more abundant outdoors (Fig. 6B).

We also evaluated the indoor and outdoor fungal profile of dry and wet (water-damage + flooded) homes (Fig. 7). Beta-diversity analysis using PCoA showed differences in the fungal communities of dry homes grouped by sampling location (indoor and outdoor) and sampling period (first and second) (Fig. 7A). No differences in group clustering were identified in samples from wet homes (Fig. 7B). Results from alpha-diversity analysis-using the Chao1 richness indicated no significant differences ( $\mathrm{p}$-value $=0.1288$ ) in the number of unique taxa in the dry homes (Fig. 7C, Supplementary Table 2) and a significant increase in the taxa present in the indoor samples from the second sampling period in wet homes (p-value $=0.002)$ (Fig. 7D). Aspergillus spp., Cladosporium spp., non-sporulating fungi and Penicillium spp. were the most abundant fungi present in the indoor environment of both dry and wet homes during both sampling periods (Fig. 8). Non-sporulating fungi and Penicillium spp. abundance inside dry homes decreased whereas Aspergillus spp. and Cladosporium spp. abundance increased during the second sampling period when compared to the first sampling period (Fig. 8A and 8C). The same trend was observed for wet homes (Fig. 8B and 8D). Aspergillus spp. and non-sporulating fungi were identified as biomarkers of dry homes, both with an FDR-adjusted p-value $=0.005$ (Fig. 9). Aspergillus spp. and non-sporulating fungi were more abundant in the indoor and outdoor environments of the second and first sampling periods, respectively (Fig. 9A and 9B).

\section{DISCUSSION}

374

375

376

Fungal quantification and fungal community characterization after hurricanes are important to develop guidelines for post-flood recovery efforts. In this work, we analyzed culturable fungal levels in homes located within a neighborhood in San Juan (PR) affected by Hurricane María's extensive flooding. Culturable fungi levels (CFU/ $\mathrm{m}^{3}$ ) were higher inside flooded homes 22 months following Hurricane María as compared to the levels measured inside 
377 dry non-flooded homes (Fig. 4B). This result is consistent with previous studies conducted in the 378 aftermath of hurricanes which have typically found higher fungal growth and fungal components

379 (spores, endotoxins and glucans) inside homes and areas that sustained moderate to severe water380 damage and/or flooding (Chew et al., 2006; Riggs et al., 2008; Barbeau et al., 2010). This rise in

381 fungal concentrations could be explained by the fact that water damage and flooding may

382 increase humidity and moisture levels inside the affected home, including wetting furniture and

383 household construction materials, thus creating favorable conditions for fungal growth indoors.

384

Since humidity is a contributing factor for fungal proliferation, we measured relative humidity levels in the homes during the different sampling periods (first and second). In terms of humidity,almost two years after Hurricane María we found higher relative humidity levels in inside flooded homes; however, this trend was not evident during the first year after Hurricane María (Fig. 2A and 2B). The ground and surrounding areas of flooded homes were inundated, during and after Hurricane María by rain and phreatic water (underground water). Although not measured through in this research, this could have also impacted relative humidity levels in the flooded homes that are also located in shallow water table areas. During the first sampling period, no differences in relative humidity levels were observed possibly due to closeness of the sampling to the hurricane and flooding events.

We analyzed our culture-based data using different tools typically applied to molecularbased data. These analyses and visualizations provided additional insight into the fungal communities present in our samples as a function of different variables (degree of water damage, indoor versus outdoor sampling location). We found significant differences in fungal composition and structure when analyzing beta-diversity by self-reported degree of water exposure during the first sampling period (p-value $<0.001$ ), meaning that the distribution of the fungal species recovered varied between the categories analyzed (Fig. 4C). However, there were no significant differences in species richness-according to alpha diversity (Fig. 4D). Meaning there were no differences in the number of species within the categories studied. Results from abundance taxonomic bar plots highlighted the shift from fungal communities present in the outdoor environment (typically non-sporulating fungi) to enrichment of taxa previously associated with damp environments and human disease (Fig. 5A and 5B). Non-sporulating fungi refer to fungi that fail to produce spores on culture media, therefore identification through microscopy techniques is not possible. Non-sporulating fungi are most likely members of the Basidiomycota phylum, which compose approximately $60 \%$ of the fungal spores present in the outdoor air of PR (Rivera-Mariani et al.; AAAAI, 2021). Thus, the indoor environment of dry non-flooded homes appears to reflect the outdoor environment (Fig. 5A). This result was expected, since the study homes, as with most homes in PR, are naturally ventilated allowing for fungal spores present in the outdoor air to enter the homes through doors and windows. In contrast, water-damage homes yielded an increase in abundance of Aspergillus taxa which eventually dominated the indoor environment of flooded homes (Fig. 5A and 5C). Approximately 180 species of Aspergillus exist, with $A$. fumigatus, $A$. terreus, $A$. niger, $A$. flavus being the most frequent causative agents of human fungal infection, including aspergillosis, asthma and allergic rhinitis (Sugui et al., 2014; 
419 Seyedmousavi et al., 2018). Moreover, Aspergillus taxa have been previously associated with 420 damp environments (Miller, Haisley \& Reinhardt, 2000; Chew et al., 2006; Solomon et al., 2006; 421 Rao et al., 2007; Schwab et al., 2007; Riggs et al., 2008; Bolaños-Rosero et al., 2013; Flores et 422 al., 2013). The shift in fungal taxa from the first to the second sampling period in water-damage 423 and flooded homes (from Aspergillus species to non-sporulating fungi predominance) indicates 424 that 22 months after Hurricane María, there has been a recovery in the fungal communities to the 425 taxa normally present in the tropical environment of Puerto Rico (Fig. 5B). Rhodotorula was 426 found significantly enriched in the outdoor environment of flooded homes (Fig. 5C). This 427 unicellular red-orange pigmented yeast is part of the Basidiomycota division and has been previously associated with water-damaged materials and moisture damage in buildings (Andersen et al., 2011; Adams et al., 2020).

Finally, the most abundant fungi recovered during the first sampling period from both the indoor and outdoor environments of dry and wet homes were Aspergillus, Cladosporium, Penicillium and non-sporulating fungi. The first three fungi correspond with findings of other studies, while non-sporulating fungi represent the characteristic fungi from the tropical outdoor environment of PR (Fig. 6A, 8 and 9) (Miller, Haisley \& Reinhardt, 2000; Chew et al., 2006; Solomon et al., 2006; Rao et al., 2007; Schwab et al., 2007; Riggs et al., 2008; Bolaños-Rosero et al., 2013; Flores et al., 2013).

Our study provides a unique and valuable data set. First, we evaluated fungal concentration and composition during two sampling periods one and two years in the aftermath of Hurricane María. Even one year after Hurricane María, the homes sampled had differences in fungal communities according to the degree of water damage. Almost 2 years after the hurricane, we found that fungal communities indoor began to recover and resemble the outdoor fungal communities. Most of the studies that evaluated fungal growth and spore levels after a Hurricane were carried out within 6 months after the natural disaster. Second, we collected our data through culture-based methods which allows analysis of viable fungi present in the sampled environment. We acknowledge the limitations of this approach for fungal identification, however useful information was still gained regarding indoor fungal amplification and diversity. Third, we used two different culture media for the recovery of fungal isolates, MEA and G25N allowing for the characterization of a wide range of fungi from hydrophilic to xerophilic fungi, respectively. Our results showed that $\mathrm{G} 25 \mathrm{~N}$ is more useful for the collection of fungal isolates that usually amplify (indoor concentrations greater than the outdoors) under damped or flooded conditions. Since G25N allows slow and discrete colony growth, all fungi can grow without a dominating genus overgrowth occupying the culture plate surface as it happens in MEA. However, the use of both culture media is recommended to recover as many fungal species as possible. We also considered the levels of outdoor fungal spores reported by the San Juan AAAAI station, which were highest during the first sampling period and contributed to the indoor fungal concentration, agreeing with an outdoor-indoor air continuum. Also, the fungal isolates obtained from these samples agreed 
461 additional insights into fungal communities' composition, richness, abundance and biomarkers 462 significantly enriched in our samples. By applying tools originally develop for data generated by 463 molecular biology approaches for the analysis of culture-based data, we able to further 464 understand our results and identify fungal features characteristic between dry and flooded homes. 465 Particularly, we ble to identify a significant shift from Aspergillus taxa to non-sporulating 466 fungi inside flooded homes between the first and second sampling period.

In summary, this data highlights dynamic changes in indoor fungal populations, characterized by different types of fungi between dry and flooded homes. More specifically, dry homes were dominated by fungi present in the outdoor environment highlighting the naturally ventilated types of homes common in PR. On the other hand, flooded homes presented amplification of Aspergillus species. Additionally, our study results indicate that in naturally ventilated homes, the outdoor fungal levels have a significant effect on indoor fungal levels. This brings attention to the outdoor environment. Thus, after-flood cleanup and remediation at a community scale may help address this issue. Since tropical homes are characterized by an outdoor-indoor air continuum (Fig. 3A and 3B), it is extremely important to maintain clean outdoor surroundings after the disaster. In this study, we observed high quantities of waterdamage materials, wood and trees debris outside many of the homes sampled. These organic materials could have also contributed to outdoor filamentous fungi proliferation that eventually entered the homes via open windows and/or doors. In-order to apply these suggestions in the context of low-income communities, assistance from local government is warranted. Disaster relief for flooding victims, including debris removal from the surroundings, covered-expenses home repairs, materials and/or furniture replacement, provide ${ }_{2}$ cleaning materials and basic education on flood preparedness and recovery. Furthermore, to prevent indoor fungal proliferation, it's recommended to maintain a clean indoor environment after flooding events. Building problems should be repaired and water-damaged materials must be cleaned, dried and/or removed. ${ }^{15}$ Studies have shown that remediation of mold-damaged materials, through cleaning or replacement, decrease or eliminate_ mold growth (Solomon et al., 2006; Riggs et al., 2008; Jayaprakash et al., 2017). It is important to identify indoor moisture sources such as roof, walls and plumbing leaks, condensation, floods and high relative humidity. An additional recommendation is to keep indoor relative humidity levels below $60 \%$, ideally between $30 \%$ and $50 \%$, in order to inhibit mold growth(EPA). Moreover, dehumidifiers and High-Efficiency Particulate Air (HEPA) filters are tools that help control fungal growth and spore levels, respectively (Mazur \& Kim, 2006). These findings are helpful for developing guidelines for postflood recovery efforts as well as strategies to mitigate respiratory diseases in tropical environment.

\section{ACKNOWLEDGEMENTS}

The authors want to thank all the families participating in this research. Additionally, we thank the team of research students that participated in this project. This research project is in partial fulfilment of Lorraine N. Vélez-Torres doctoral thesis dissertation. 


\section{REFERENCES}

AAAAI. 2021.National Allergy Bureau (NAB): Pollen and Mold Levels San Juan Station. Available at https://pollen.aaaai.org/nab/index.cfm?p=allergenreport\&stationid=168\#/station/39ad34be-177c-47dd-acd799b67d1e2afb/calendar/2021/12 (accessed 2021).

Adams RI, Sylvain I, Spilak MP, Taylor JW, Waring MS, Mendell MJ. 2020. Fungal Signature of Moisture Damage in Buildings: Identification by Targeted and Untargeted Approaches with Mycobiome Data. Applied and Environmental Microbiology 86-DOI: 10.1128/aem.01047-20.

Andersen B, Frisvad JC, Søndergaard I, Rasmussen IS, Larsen LS. 2011. Associations between Fungal Species and Water-Damaged Building Materials. Applied and Environmental Microbiology 77:4180-4188. DOI: 10.1128/aem.02513-10.

Arndt D, Xia J, Liu Y, Zhou Y, Guo AC, Cruz JA, Sinelnikov I, Budwill K, Nesbø CL, Wishart DS. 2012. METAGENassist: a comprehensive web server for comparative metagenomics. Nucleic Acids Research 40:W88-W95. DOI: 10.1093/nar/gks497.

Barbeau DN, Grimsley LF, White LE, El-Dahr JM, Lichtveld M. 2010. Mold Exposure and Health Effects Following Hurricanes Katrina and Rita. Annual Review of Public Health 31:165-178. DOI: 10.1146/annurev.publhealth.012809.103643.

Bolaños-Rosero B. 2021. Mold and Pollen Levels in San Juan, Puerto Rico: Trends Observed Over 15 Years of Research. Unpublished Manuscript.

Bolaños-Rosero B, Betancourt D, Dean T, Vesper S. 2013. Pilot study of mold populations inside and outside of Puerto Rican residences. Aerobiologia 29:537-543. DOI: 10.1007/s10453-013-9301-7.

Cannon P, Aguirre-Hudson B, Aime M, Ainsworth A, Bidartondo M, Gaya E, Hawksworth D, Kirk P, Leitch I, Lücking R. 2018. Definition and diversity. In: Willis KJ ed. State of the World's Fungi 2018. Kew: Royal Botanic Gardens, 4-11.

(CDC) Centers for Disease Control and Prevention. 2006. Health concerns associated with mold in water-damaged homes after Hurricanes Katrina and Rita--New Orleans area, Louisiana, October 2005. Morbidity \& Mortality Weekly Report 2:41-44.

Chew GL, Wilson J, Rabito FA, Grimsley F, Iqbal S, Reponen T, Muilenberg ML, Thorne PS, Dearborn DG, Morley RL. 2006. Mold and Endotoxin Levels in the Aftermath of Hurricane Katrina: A Pilot Project of Homes in New Orleans Undergoing Renovation. Environmental Health Perspectives 114:1883-1889. DOI: 10.1289/ehp.9258.

Chow NA, Toda M, Pennington AF, Anassi E, Atmar RL, Cox-Ganser JM, Silva JD, Garcia B, Kontoyiannis DP, Ostrosky-Zeichner L, Leining LM, McCarty J, Mohajer MA, Murthy BP, Park J-H, Schulte J, Shuford JA, Skrobarcek KA, Solomon S, Strysko J, Chiller TM, Jackson BR, Chew GL, Beer KD. 2019. HurricaneAssociated Mold Exposures Among Patients at Risk for Invasive Mold Infections After Hurricane Harvey Houston, Texas, 2017. Morbidity and Mortality Weekly Report 68:469-473. DOI: 10.15585/mmwr.mm6821a1.

Claub M. 2015. Particle size distribution of airborne micro- organisms in the environment â€“ a review. Applied Agricultural and Forestry Research 65:77-100. DOI: 10.3220/lbf1444216736000.

De León JG, Méndez RG, Cadilla C, Rivera-Mariani FE, Bolaños-Rosero B. 2018. Identification of Immunoglobulin E-Binding Proteins of the Xerophilic Fungus Aspergillus penicillioides Crude Mycelial Mat Extract and Serological Reactivity Assessment in Subjects with Different Allergen Reactivity Profiles. International Archives of Allergy and Immunology:147-159. DOI: doi.org/10.1159/000484898. 
546

547

548

549

550

551

552

553

554

555

556

557

558

559

560

561

562

563

564

565

566

567

568

569

570

571

572

573
Dhariwal A, Chong J, Habib S, King IL, Agellon LB, Xia J. 2017. MicrobiomeAnalyst: a web-based tool for comprehensive statistical, visual and meta-analysis of microbiome data. Nucleic Acids Research 45:W180W188. DOI: 10.1093/nar/gkx295.

EPA. A Brief Guide to Mold, Moisture and Your Home. Available at https://www.epa.gov/mold/brief-guide-moldmoisture-and-your-home (accessed November 10, 2021).

Flores MEB, Medina PG, Camacho SPD, Beltrán M de JU, Otero M del CD la C, Ramírez IO, Hernández MET. 2013. Fungal spore concentrations in indoor and outdoor air in university libraries, and their variations in response to changes in meteorological variables. International Journal of Environmental Health Research 24:320-340. DOI: 10.1080/09603123.2013.835029.

Gargano LM, Locke S, Jordan HT, Brackbill RM. 2018. Lower Respiratory Symptoms Associated With Environmental and Reconstruction Exposures After Hurricane Sandy. Disaster Medicine and Public Health Preparedness 12:697-702. DOI: 10.1017/dmp.2017.140.

Gautier C, Charpin D. 2017. Environmental triggers and avoidance in the management of asthma. Journal of Asthma and Allergy Volume10:47-56. DOI: 10.2147/jaa.s121276.

Global Asthma Network. 2018. The Global Asthma Report: 2014. Auckland New Zeland.

Hamilos DL. 2010. Allergic Fungal Rhinitis and Rhinosinusitis. Proceedings of the American Thoracic Society 7:245-252. DOI: 10.1513/pats.200909-098al.

Institute of Medicine. 2004. Damp Indoor Spaces and Health. Washington (DC): National Academies Press (US).

Intellectus Statistics [Online computer software]. https://www.intellectusstatistics.com

Jayaprakash B, Adams RI, Kirjavainen P, Karvonen A, Vepsäläinen A, Valkonen M, Järvi K, Sulyok M, Pekkanen J, Hyvärinen A, Täubel M. 2017. Indoor microbiota in severely moisture damaged homes and the impact of interventions. Microbiome 5:138. DOI: 10.1186/s40168-017-0356-5.

Joubert AI, Geppert M, Johnson L, Mills-Goodlet R, Michelini S, Korotchenko E, Duschl A, Weiss R, Horejs-Höck J, Himly M. 2020. Mechanisms of Particles in Sensitization, Effector Function and Therapy of Allergic Disease. Frontiers in Immunology 11:1334. DOI: 10.3389/fimmu.2020.01334.

Kendrick B. 2011. Fungi: Ecological Importance and Impact on Humans. eLS. DOI: 10.1002/9780470015902.a0000369.pub2.

Kołodziejczyk K, Bozek A. 2016. Clinical Distinctness of Allergic Rhinitis in Patients with Allergy to Molds. BioMed Research International 2016:1-6. DOI: 10.1155/2016/3171594.

Kontoyiannis DP, Shah EC, Wurster S, Aitken SL, Graviss L, Raad II, Chemaly RF. 2019. Culture-documented invasive mold infections at MD Anderson Cancer Center in Houston, Texas pre and post hurricane Harvey. Open Forum Infectious Diseases 6:ofz138-. DOI: 10.1093/ofid/ofz138.

Kwon-Chung KJ, Sugui JA. 2013. Aspergillus fumigatus-What Makes the Species a Ubiquitous Human Fungal Pathogen? PLoS Pathogens 9:e1003743. DOI: 10.1371/journal.ppat.1003743.

Lewis LM, Mirabelli MC, Beavers SF, Kennedy CM, Shriber J, Stearns D, González JJM, Santiago MS, Félix IM, Ruiz-Serrano K, Dirlikov E, Lozier MJ, Sircar K, Flanders WD, Rivera-García B, Irizarry-Ramos J, BolañosRosero B. 2019. Characterizing environmental asthma triggers and healthcare use patterns in Puerto Rico. Journal of Asthma:1-12. DOI: 10.1080/02770903.2019.1612907. 
Li CH, Cervantes M, Springer DJ, Boekhout T, Ruiz-Vazquez RM, Torres-Martinez SR, Heitman J, Lee SC. 2011. Sporangiospore Size Dimorphism Is Linked to Virulence of Mucor circinelloides. PLoS Pathogens 7:e1002086. DOI: 10.1371/journal.ppat.1002086.

Macher J. 1989. Positive-hole correction of multiple-jet impactors for collecting viable microorganisms. American Industrial Hygiene Association Journal 11:561-8. DOI: 10.1080/15298668991375164.

Mazur L, Kim J. 2006. Spectrum of noninfectious health effects from molds. Pediatrics 6:2582-6.

Miller JD, Haisley PD, Reinhardt JH. 2000. Air Sampling Results in Relation to Extent of Fungal Colonization of Building Materials in Some Water Damaged Buildings. Indoor Air 10:146-151. DOI: 10.1034/j.16000668.2000.010003146.x.

NWSFO. 2014. Preliminary Monthly Weather Data (CF6). San Juan Area. Available at https://w2.weather.gov/climate/index.php?wfo=sju (accessed July 31, 2019).

Ortiz-Rivera MC. 2018. Asthma-related health services and asthma control among women in Puerto Rico. SAGE Open Medicine 6:2050312117745903. DOI: 10.1177/2050312117745903.

Portnoy JM, Barnes CS, Kennedy K. 2008. Importance of Mold Allergy in Asthma. Current Allergy \& Asthma Reports 8:71-78.

Quintero E, Rivera-Mariani F, Bolaños-Rosero B. 2010. Analysis of environmental factors and their effects on fungal spores in the atmosphere of a tropical urban area (San Juan, Puerto Rico). Aerobiologia 26:113-124. DOI: 10.1007/s10453-009-9148-0.

Rabito FA, Iqbal S, Kiernan MP, Holt E, Chew GL. 2008. Children’s respiratory health and mold levels in New Orleans after Katrina: A preliminary look. Journal of Allergy and Clinical Immunology 121:622-625. DOI: 10.1016/j.jaci.2007.11.022.

Rao CY, Riggs MA, Chew GL, Muilenberg ML, Thorne PS, Sickle DV, Dunn KH, Brown C. 2007. Characterization of Airborne Molds, Endotoxins, and Glucans in Homes in New Orleans after Hurricanes Katrina and Rita $\nabla$. Applied and Environmental Microbiology 73:1630-1634. DOI: 10.1128/aem.01973-06.

Riggs MA, Rao CY, Brown CM, Sickle DV, Cummings KJ, Dunn KH, Deddens JA, Ferdinands J, Callahan D, Moolenaar RL, Pinkerton LE. 2008. Resident cleanup activities, characteristics of flood-damaged homes and airborne microbial concentrations in New Orleans, Louisiana, October 2005. Environmental Research 106:401409. DOI: 10.1016/j.envres.2007.11.004.

Rivera-Mariani FE, Almaguer M, Aira MJ, Bolaños-Rosero B. Comparison of Atmospheric Fungal Spore Concentrations between Two Main Cities in the Caribbean Basin. Puerto Rico Health Sciences Journal 39:235242.

Rivera-Mariani FE, Nazario-Jiménez S, López-Malpica F, Bolaños-Rosero B. 2011. Sensitization to airborne ascospores, basidiospores, and fungal fragments in allergic rhinitis and asthmatic subjects in San Juan, Puerto Rico. International archives of allergy and immunology 155:322-34. DOI: 10.1159/000321610.

Roswell M, Dushoff J, Winfree R. 2021. A conceptual guide to measuring species diversity. OIKOS 130:321-338.

RStudio-Team. 2020. RStudio: Integrated Development for R. Boston, MA: RStudio, PBC.

Samson R, Houbraken J, Thrane U, Frisvad J, Andersen B. 2019. Food and Indoor Fungi. Centraalbureau voor Schimmelcultures.

PeerJ reviewing PDF | (2021:10:66439:1:0:NEW 3 Dec 2021) 
622

623

624

625

626

627

628

629

630

631

632

633

634

635

636

637

638

639

640
Saporta D, Hurst D. 2017. Increased Sensitization to Mold Allergens Measured by Intradermal Skin Testing following Hurricanes. Journal of Environmental and Public Health 2017:1-5. DOI: 10.1155/2017/2793820.

Schwab KJ, Gibson KE, Williams DL, Kulbicki KM, Lo CP, Mihalic JN, Breysse PN, Curriero FC, Geyh AS. 2007. Microbial and Chemical Assessment of Regions within New Orleans, LA Impacted by Hurricane Katrina. Environmental Science \& Technology 41:2401-2406. DOI: 10.1021/es062916x.

Segata N, Izard J, Walron L, Gevers D, Miropolsky L, Garrett W, Huttenhower C. 2011. Metagenomic Biomarker Discovery and Explanation. Genome Biology 12. DOI: 10.1186/gb-2011-12-6-r60.

Seyedmousavi S, Lionakis MS, Parta M, Peterson SW, Kwon-Chung KJ. 2018. Emerging Aspergillus Species Almost Exclusively Associated With Primary Immunodeficiencies. Open Forum Infectious Diseases 5:ofy213-. DOI: $10.1093 /$ ofid/ofy213.

Solomon GM, Hjelmroos-Koski M, Rotkin-Ellman M, Hammond SK. 2006. Airborne Mold and Endotoxin Concentrations in New Orleans, Louisiana, after Flooding, October through November 2005. Environmental Health Perspectives 114:1381-1386. DOI: 10.1289/ehp.9198.

Sugui JA, Kwon-Chung KJ, Juvvadi PR, Latge J-P, Steinbach WJ. 2014. Aspergillus fumigatus and Related Species. Cold Spring Harbor Perspectives in Medicine 5:a019786-a019786. DOI: 10.1101/cshperspect.a019786.

WHO, Regional Office for Europe. 2009. WHO Guidelines for Indoor Air Quality: Dampness and Mould.

Wu PC, Su HJ, Ho HM. 2000. A comparison of sampling media for environmental viable fungi collected in a hospital environment. Environmental Research 82:253-257. DOI: 10.1006/enrs.1999.4017. PMID: 10702333. 


\section{Table 1 (on next page)}

Number of homes categorized by self-reported degree of water-damage.

The total number of samples for each sampling period, total number of fungal colonies observed, and total number of unique fungal species present are summarized for each category. 
1

\begin{tabular}{cccccc}
\hline $\begin{array}{c}\text { Self-Reported } \\
\text { Degree of Water } \\
\text { Damage }\end{array}$ & Sampling Period & $\begin{array}{c}\text { Number of } \\
\text { Homes }\end{array}$ & $\begin{array}{c}\text { Number of } \\
\text { Samples }\end{array}$ & $\begin{array}{c}\text { Number of } \\
\text { Fungal Colonies }\end{array}$ & $\begin{array}{c}\text { Number of } \\
\text { Fungal Species }\end{array}$ \\
\hline \multirow{2}{*}{ Dry } & First & 13 & 124 & 4,229 & 40 \\
Water- Damage & Second & 11 & 108 & 1,925 & 33 \\
& First & 24 & 240 & 10,624 & 42 \\
Flooded & Second & 15 & 150 & 7,136 & 35 \\
\hline
\end{tabular}

2

3 
Figure 1

Map of the homes sampled in San Juan, Puerto Rico $(n=50)$.

Blue, yellow and red dots corresponds to Dry, Water-Damage and Flooded homes, respectively. 


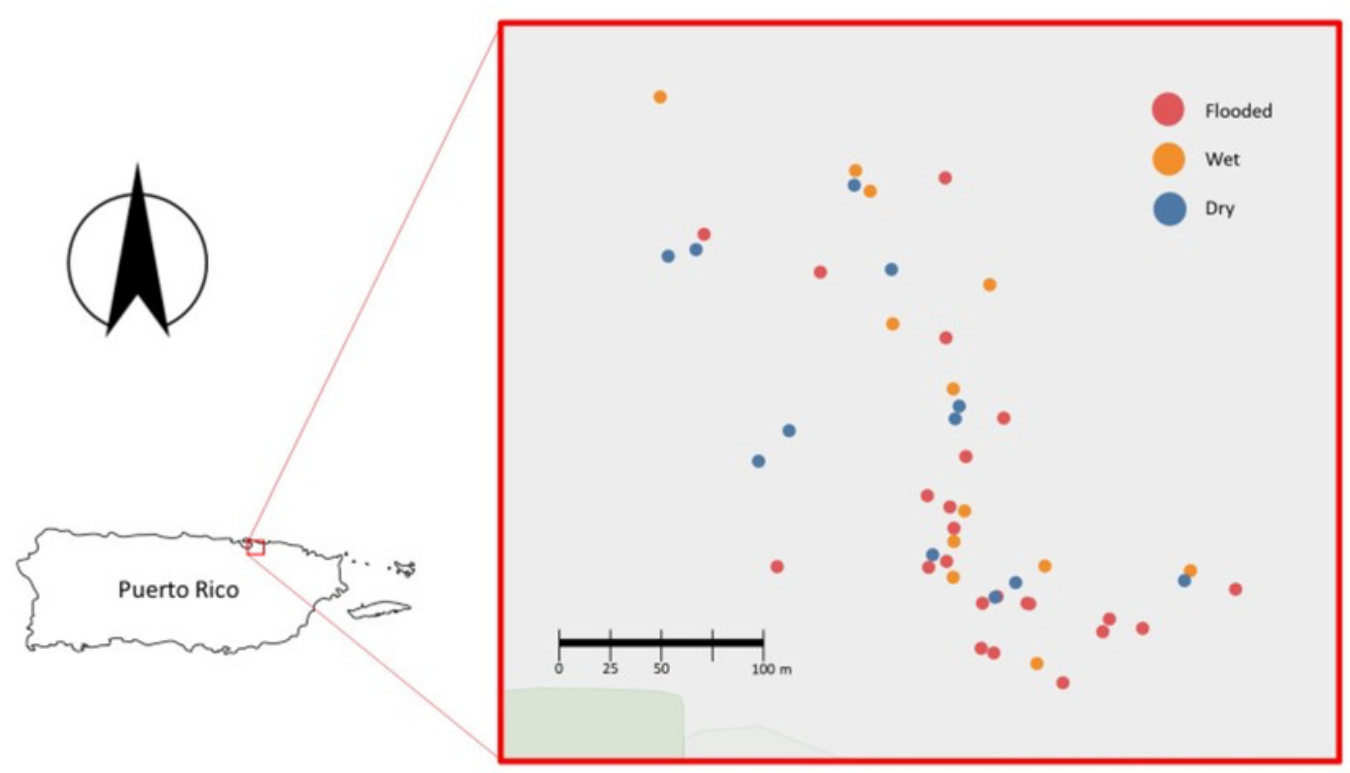


Figure 2

Indoor relative humidity levels (\%) in homes as a function of self-reported degree of water damage (Dry, Water-Damage, Flooded).

A) First Sampling ( $n=50$ homes). B) Second Sampling $(n=35)$.

cond 审 Dry

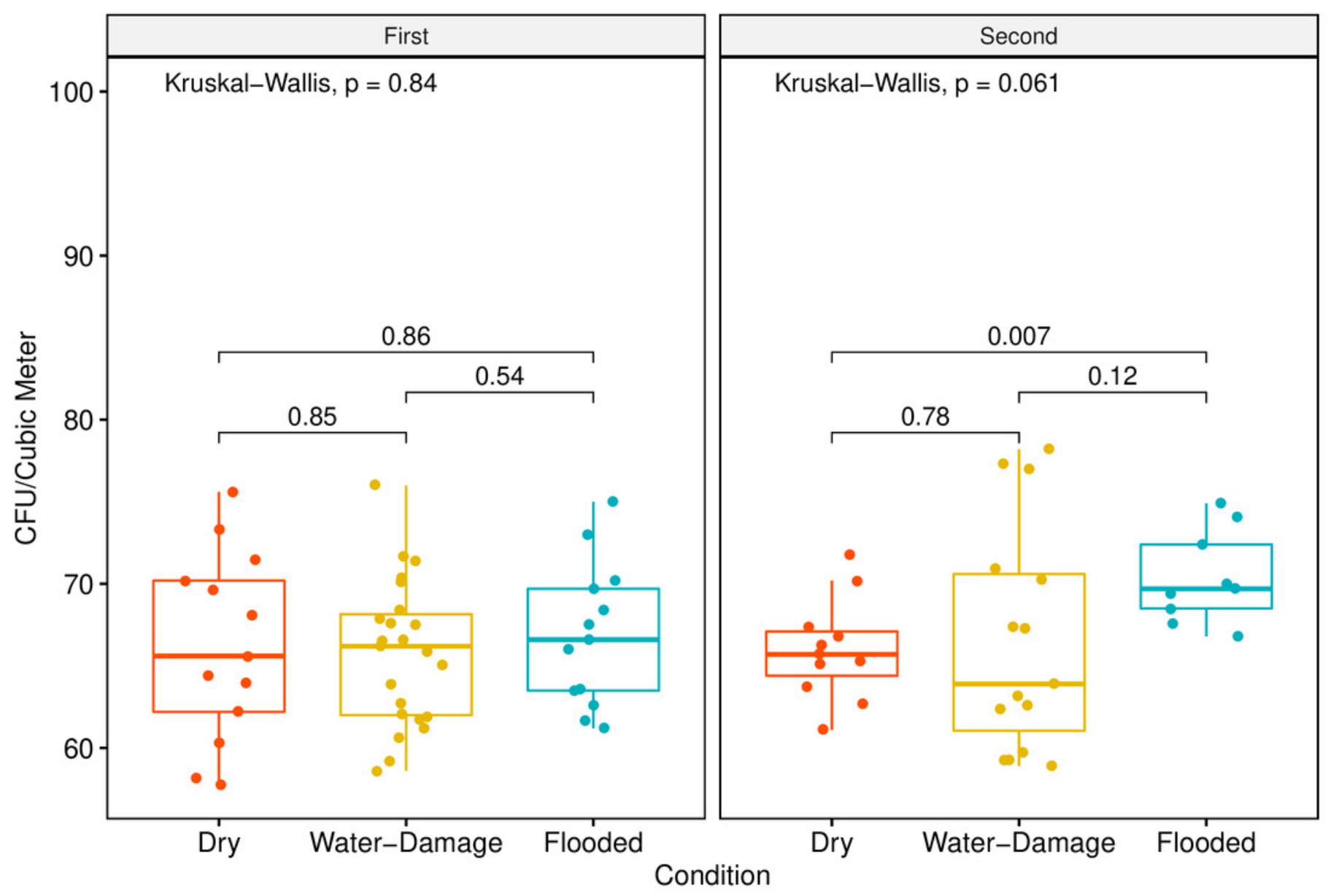


Figure 3

Linear Regression Indoor Fungal Spores and Outdoor Fungal Spores per cubic meter $\left(\mathrm{m}^{3}\right)$ of air in G25N Culture Media.

A) First sampling (2018) and B) Second sampling (2019). 
A)

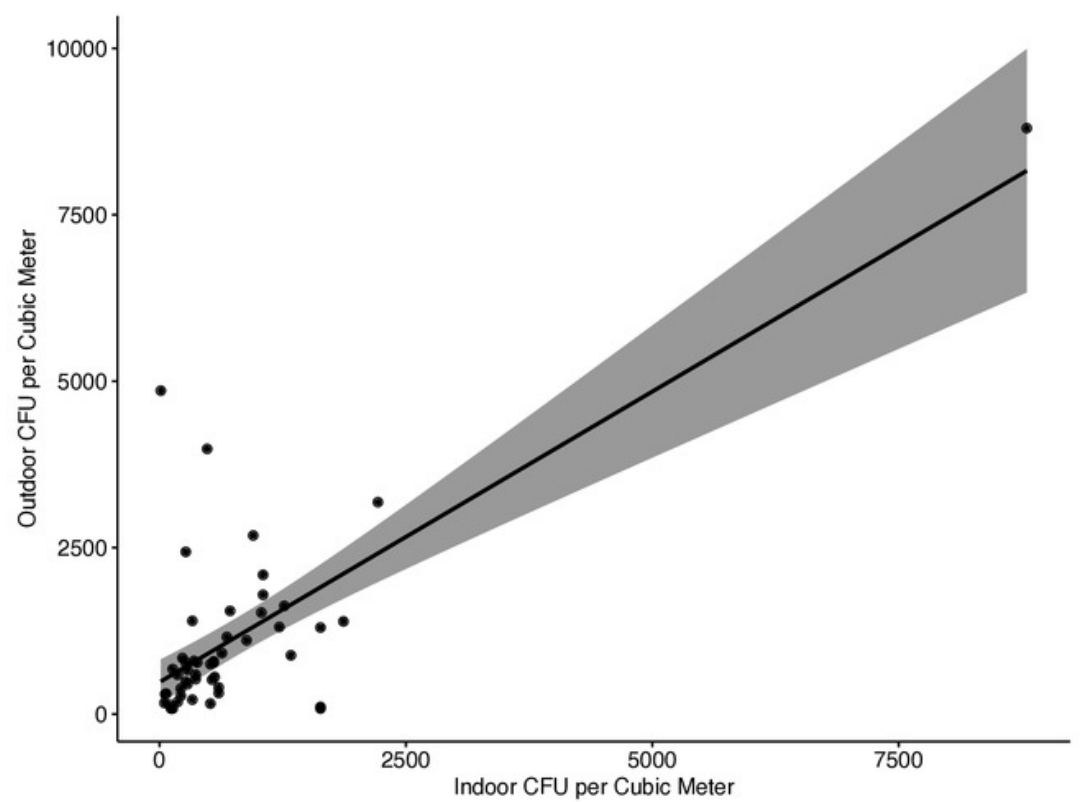

B)

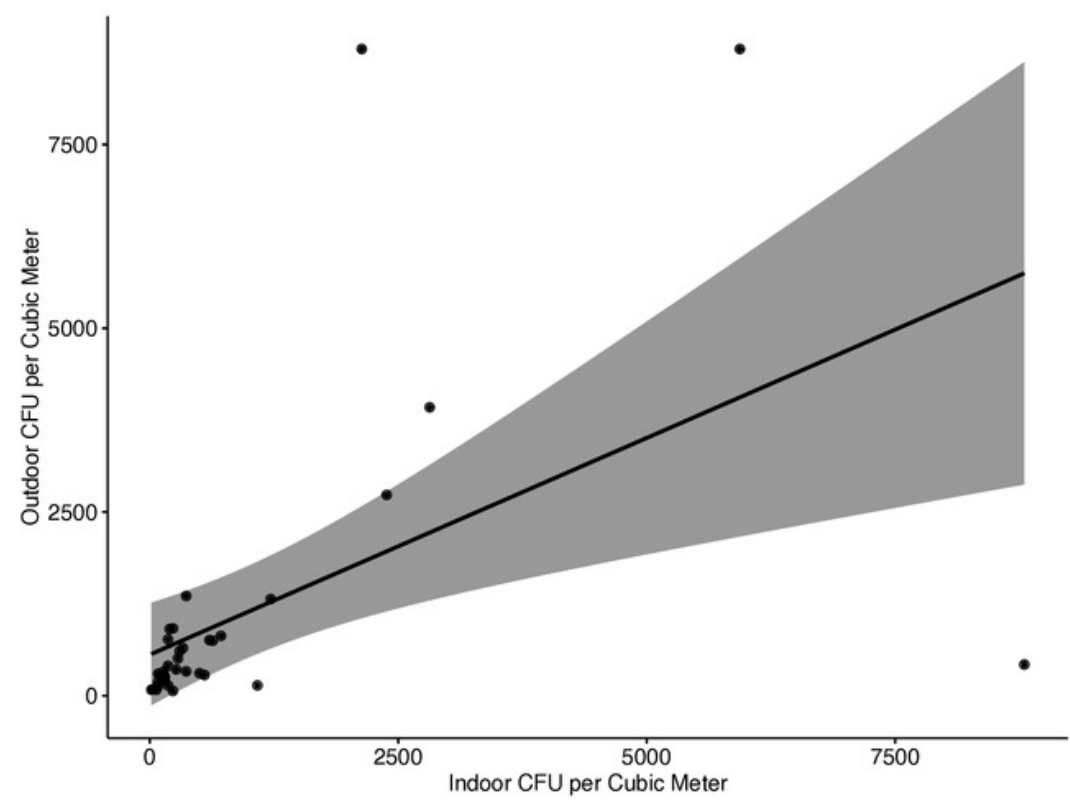




\section{Figure 4}

Fungal concentration and profile by self-reported degree of water damage (Dry, WaterDamage, Flooded).

A) G25N culture media. B) MEA culture media. C) Beta-diversity analysis for first sampling (2018). D) Alpha-diversity analysis for first sampling (2018). A, B) Red, Yellow and Blue boxplots correspond to Dry, Water-Damage and Flooded homes, respectively; first sampling $\mathrm{n}=50$ homes and second sampling $\mathrm{n}=35$ homes; Kruskal-Wallis test, $\mathrm{p}$-value $<0.05$ was considered significant. C) Principal coordinate analysis (PCOA) performed with JensenShannon Divergence. D) Chaol richness estimate. 
A)

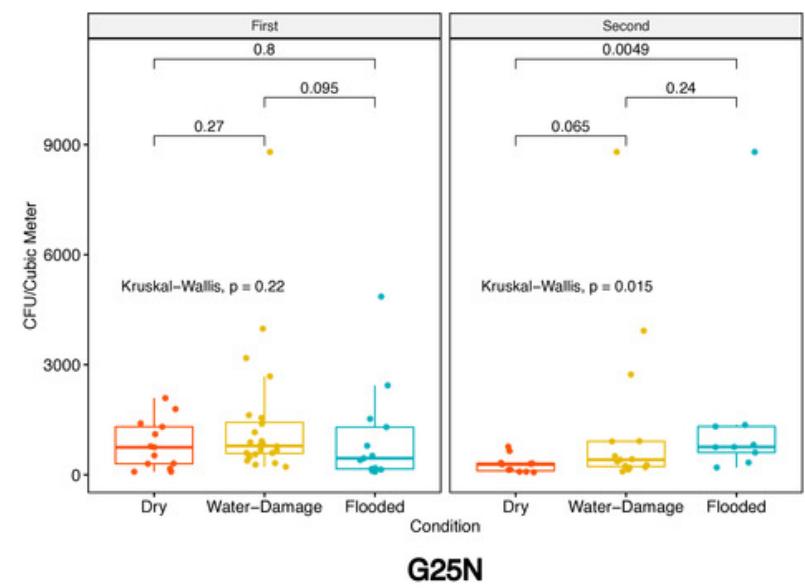

C)

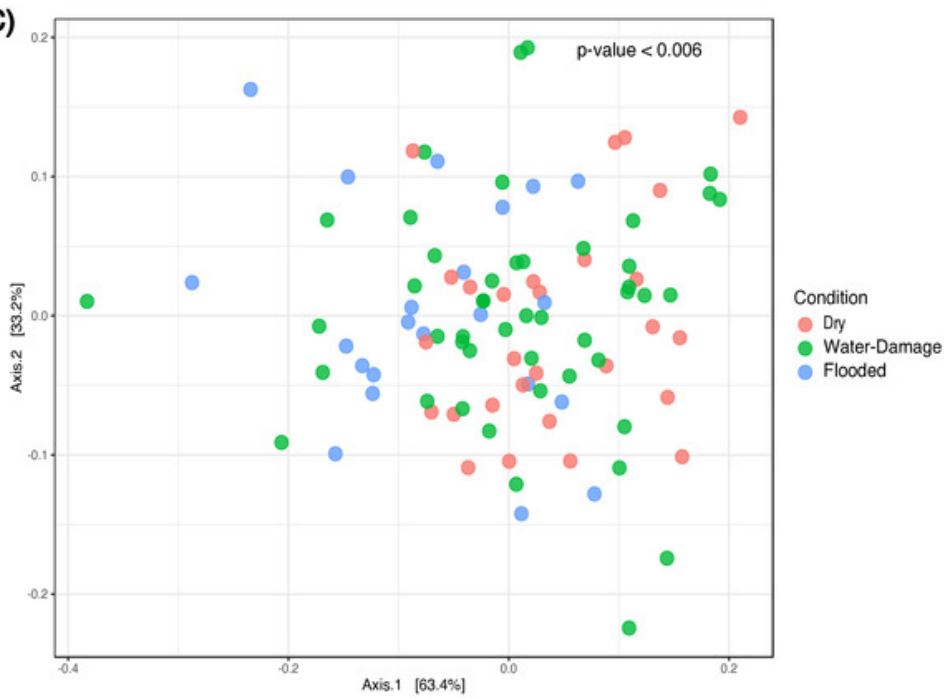

B)

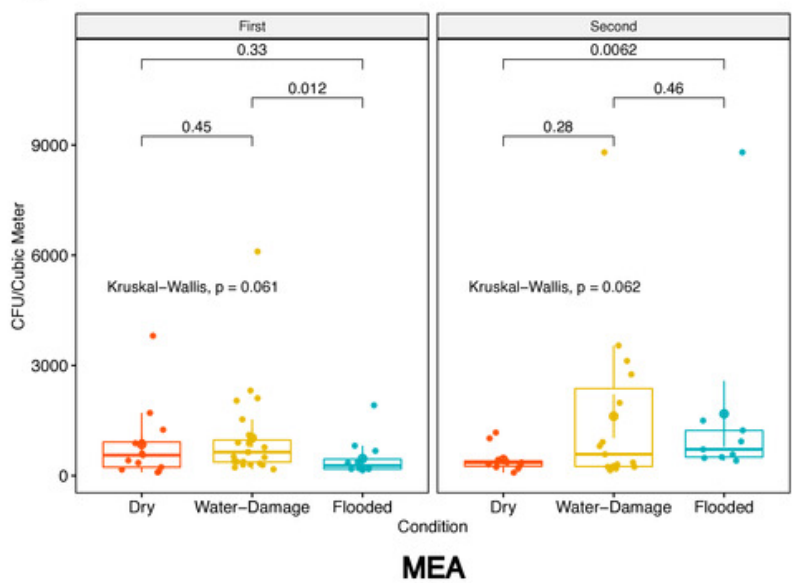

D)

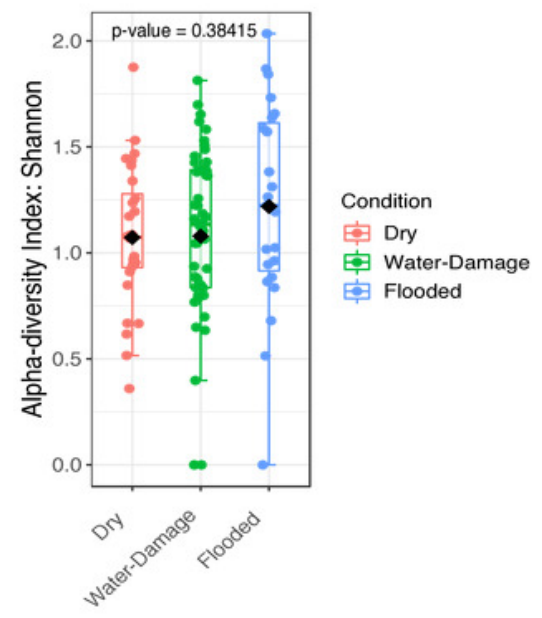




\section{Figure 5}

Abundance taxa bar plots and histogram of Linear Discriminant Analysis (LDA) Effect Size (LEfSe) by sampling location (Indoor, Outdoor), self-reported degree of waterdamage (Dry, Water-Damage, Flooded) and sampling period (First, 2018 and Second, 2019).

A) Abundance taxa bar plots during first sampling (2018). B) Abundance taxa bar plots during second sampling (2019). C) LDA during first sampling (2018), FDR-adjusted $p$-value $=0.05$. Log LDA score computed for differentially abundant taxa (genus level) with cut-off LDA score $>2.0$. 
A)

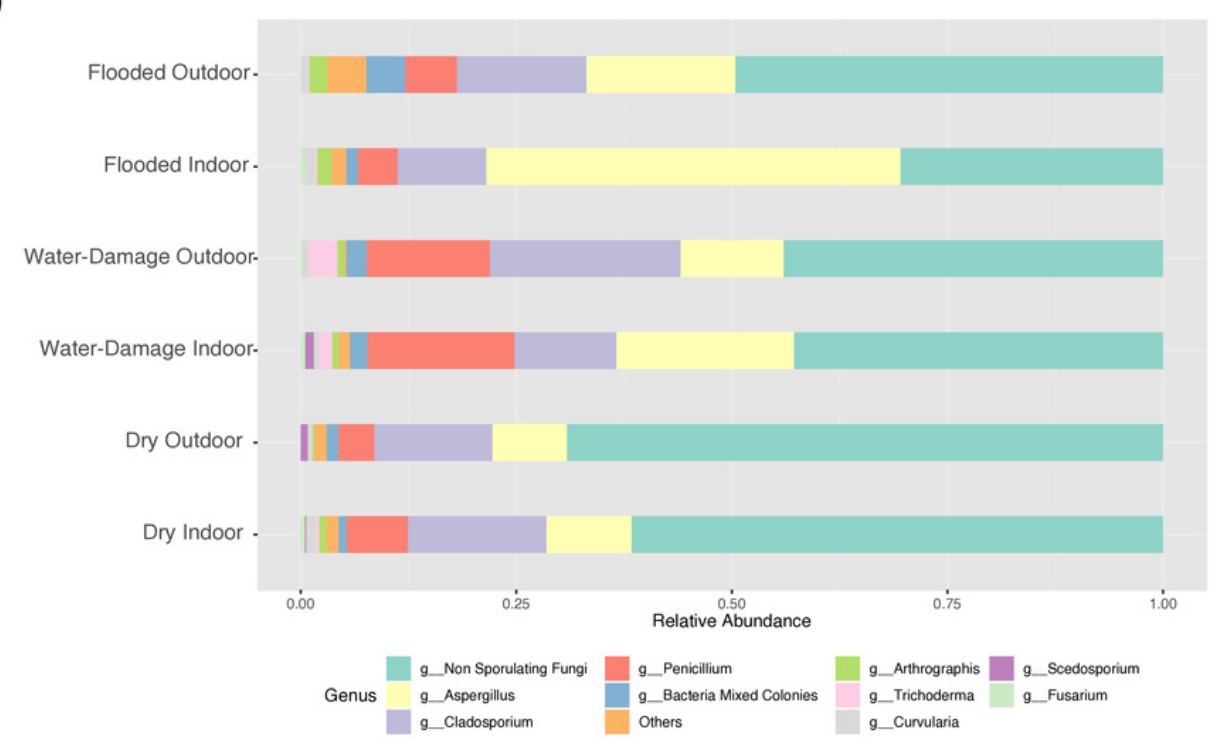

B)

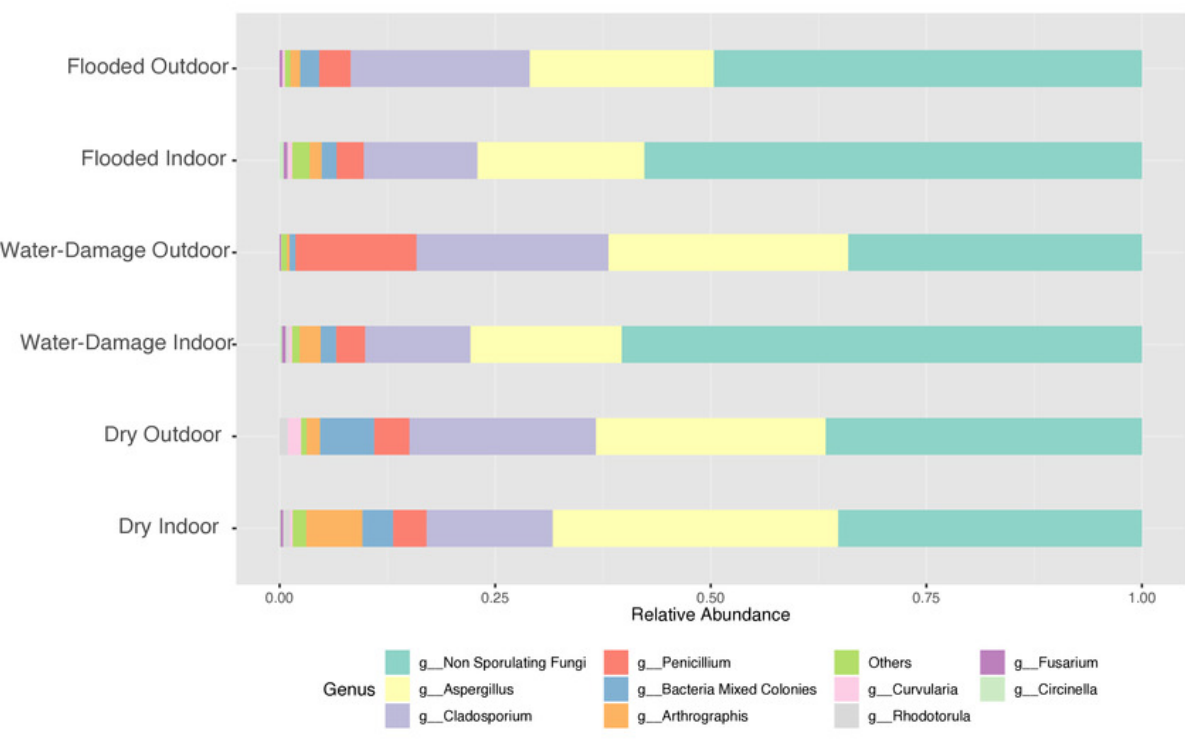

C)

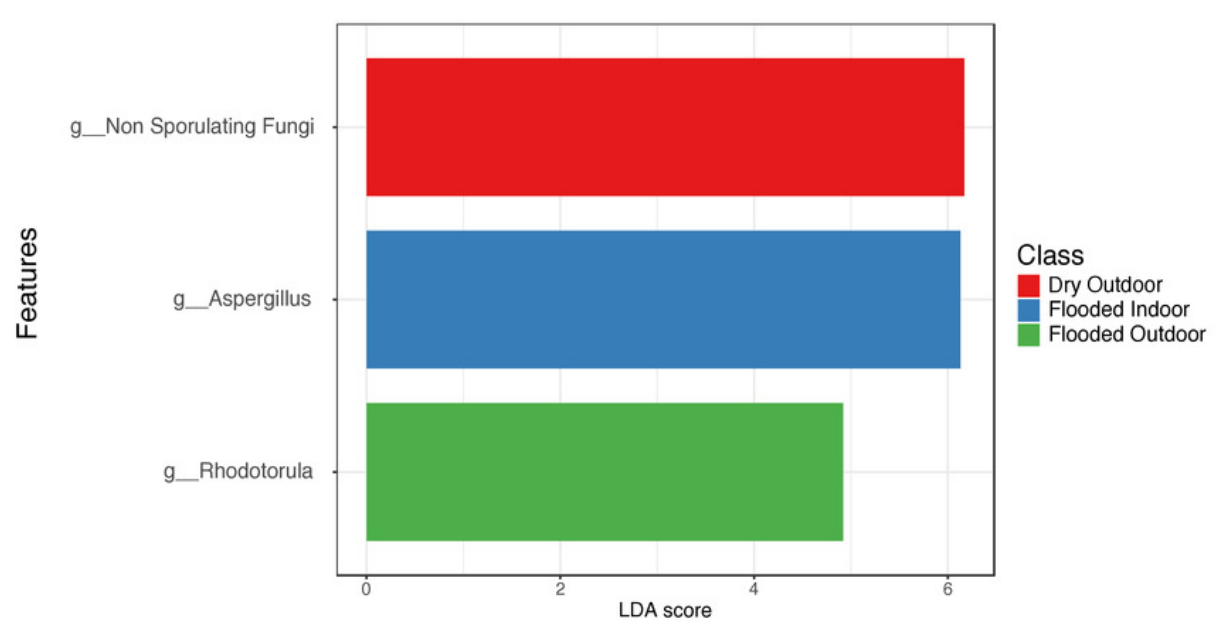




\section{Figure 6}

Individual fungi normalized abundance boxplots by sampling location (Indoor, Outdoor).

A) Individual fungi abundance boxplots during first sampling (2018). B) Individual fungi abundance boxplots during second sampling (2019). A, B) Red and Green boxplots corresponds to indoor and outdoor samples, respectively. 
A) Year 1
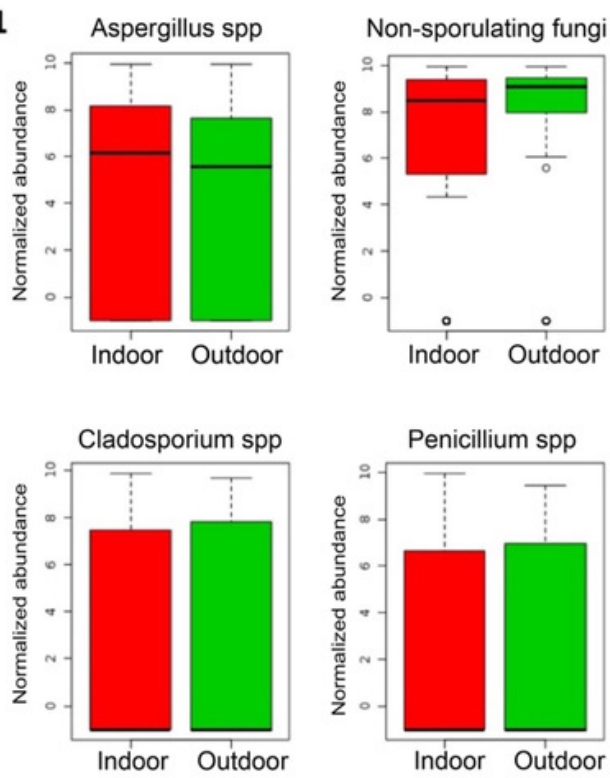

B) Year 2
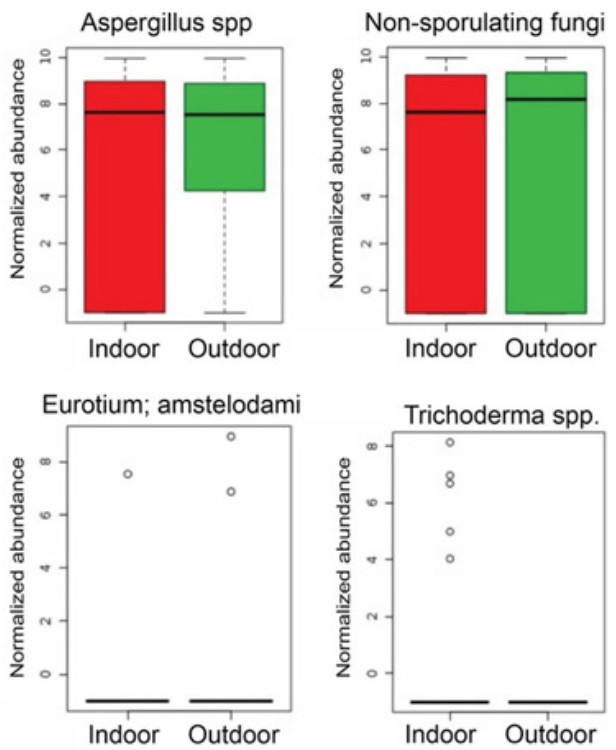


\section{Figure 7}

Fungal profile of dry and wet (Water-Damage and Flooded Homes Combined) homes during both sampling periods (First and Second).

A) Beta-diversity analysis for dry homes. B) Beta-diversity analysis for wet homes. A, B) Red, Dark Blue, Green and Light Blue dots corresponds to indoor samples from first sampling, outdoor samples for first sampling, indoor samples from second sampling and outdoor samples from second sampling; respectively. Principal coordinate analysis (PCoA) performed with Jensen-Shannon Divergence. C) Alpha-diversity analysis for dry homes. D) Alphadiversity analysis for wet homes. C, D) Red, Green, Blue and Purple boxplots corresponds to indoor samples from first sampling, indoor samples from second sampling, outdoor samples for first sampling, and outdoor samples from second sampling; respectively. Chaol richness estimate. 
A)

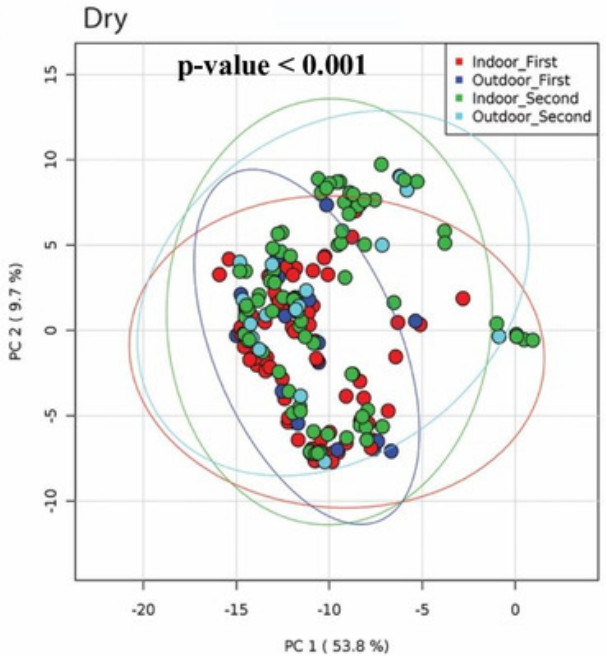

C)

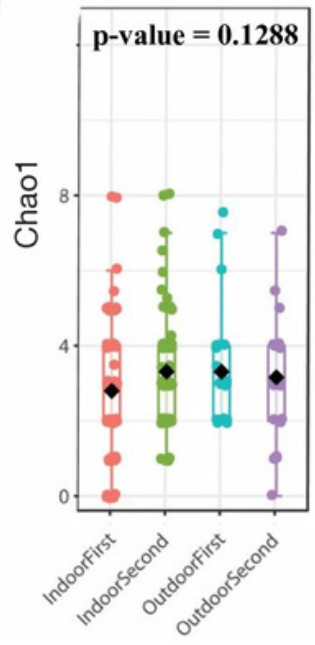

B) Wet

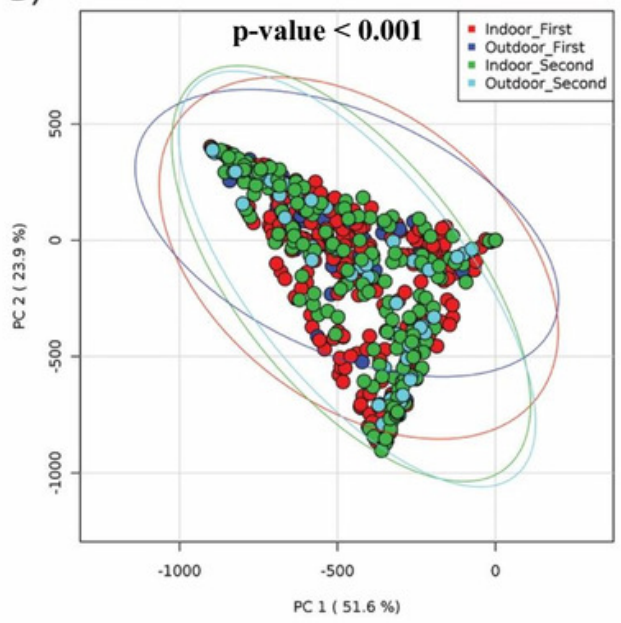

D)

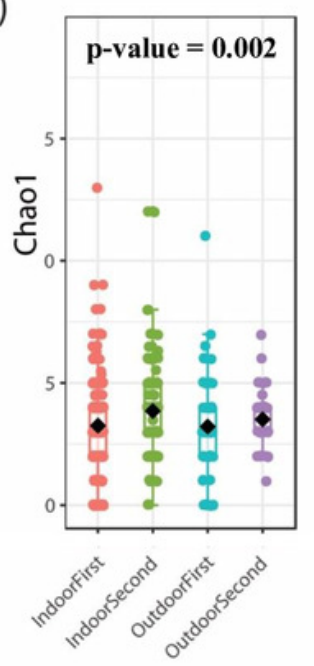




\section{Figure 8}

Indoor fungal isolates abundance percent by sampling period (First, Second).

A) Samples from indoor environment of dry homes during the first sampling period. B)

Samples from indoor environment of wet homes during the first sampling period. C) Samples from indoor environment of dry homes during the second sampling period. D) Samples from indoor environment of wet homes during the second sampling period. 

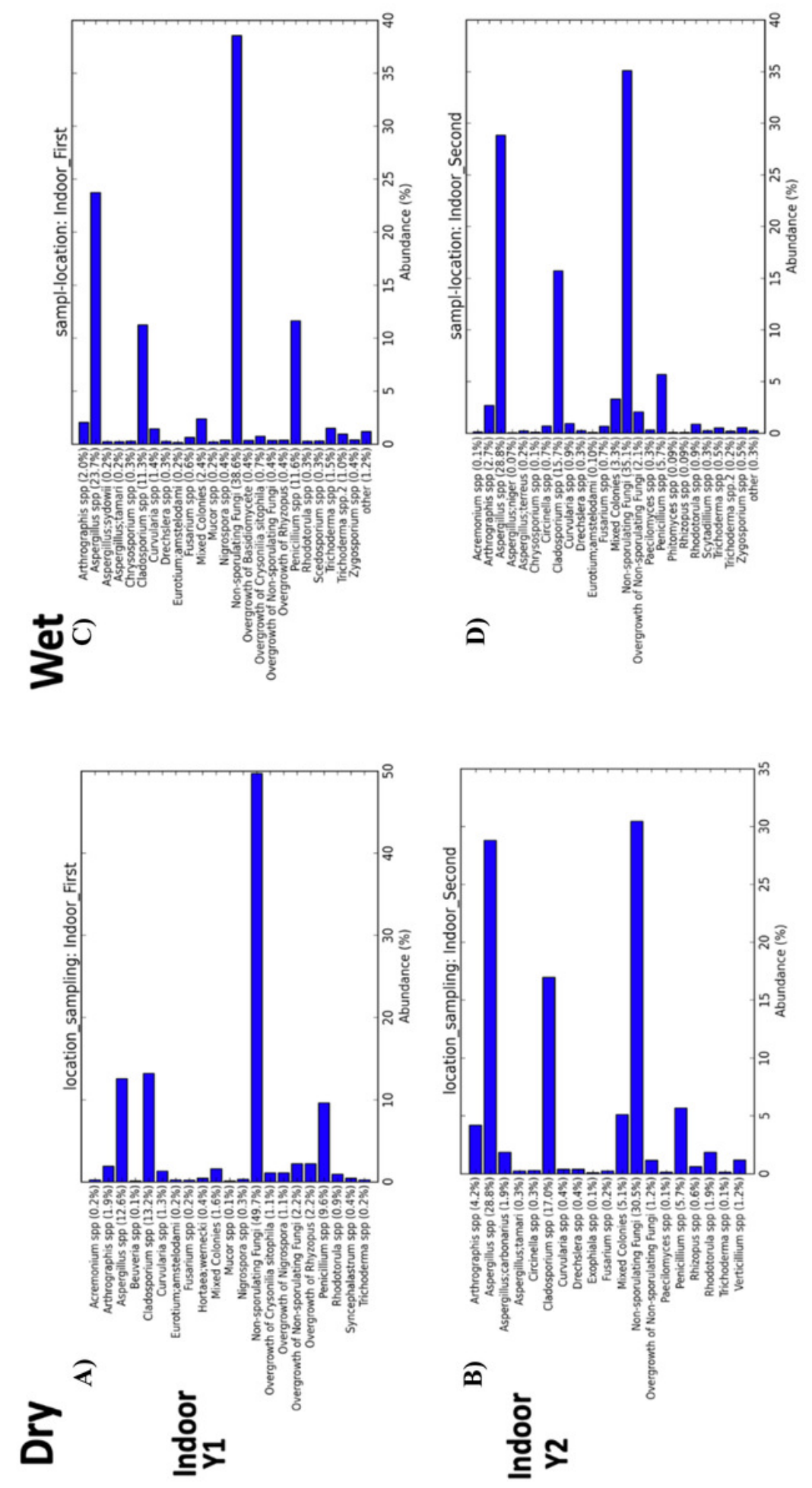
Figure 9

Fungal dry biomarkers by sampling location (Indoor, Outdoor) and sampling period (First, Second).
A) Aspergillus spp. abundance levels. B) Non-sporulating fungi abundance levels. A, B) Red, Dark Blue, Green and Light Blue boxplots corresponds to indoor samples from first sampling, indoor samples from second sampling, outdoor samples for first sampling, and outdoor samples from second sampling; respectively. 
A)

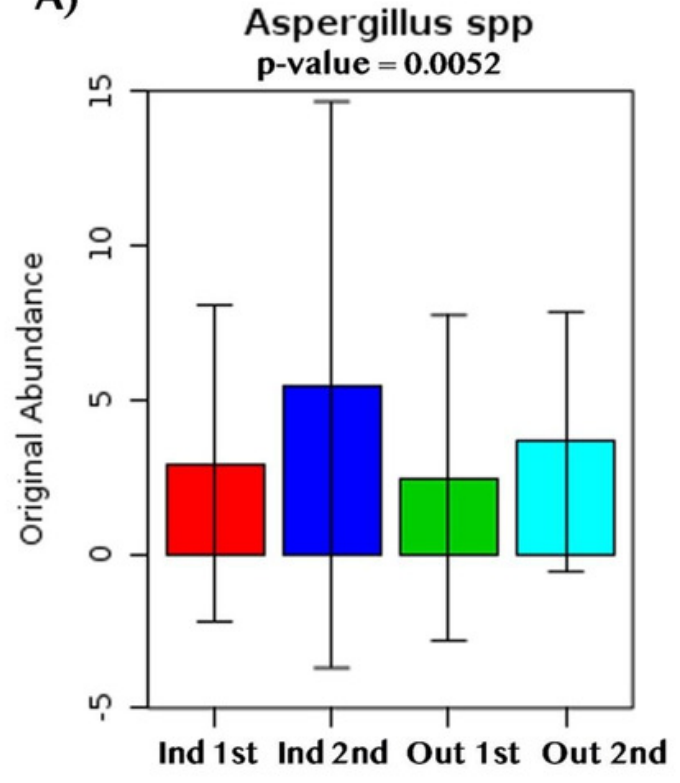

B)

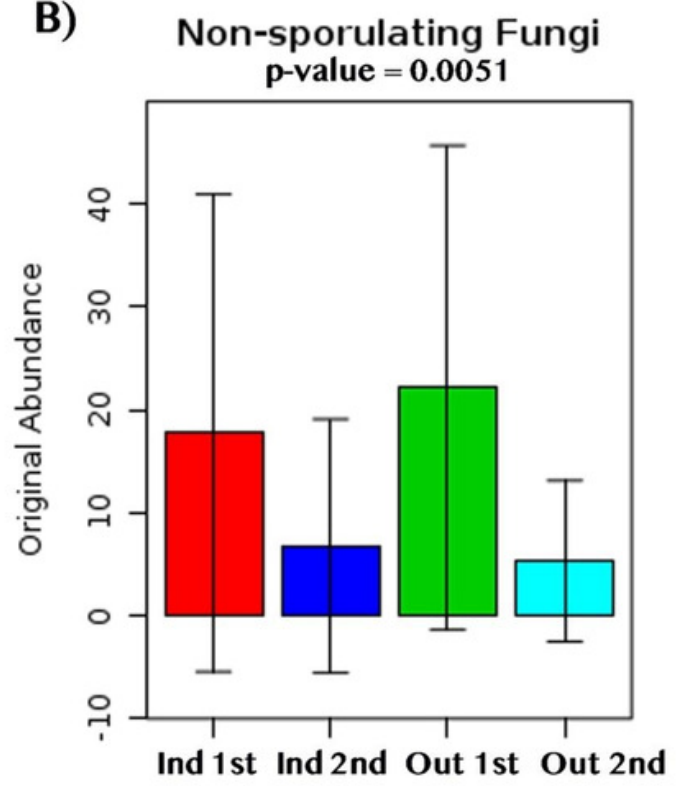

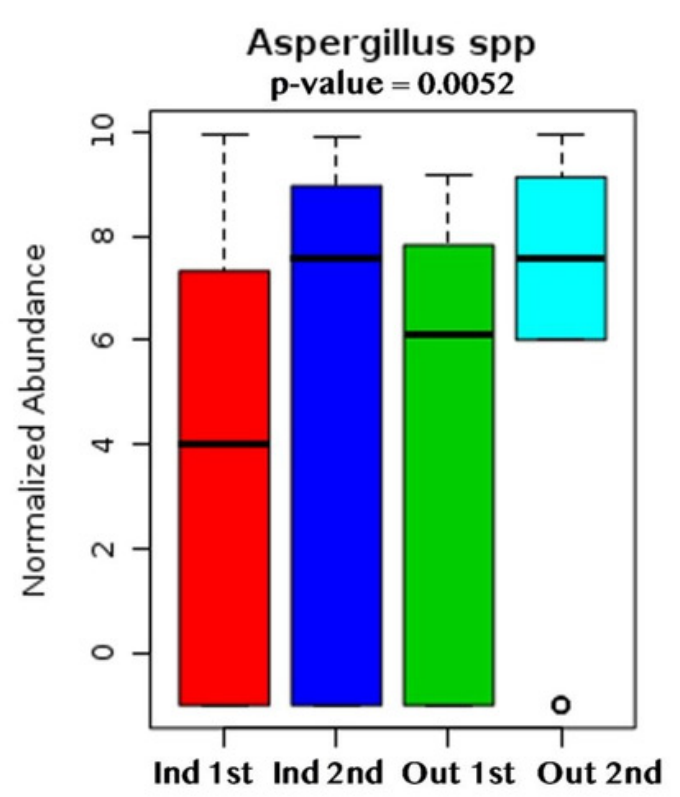

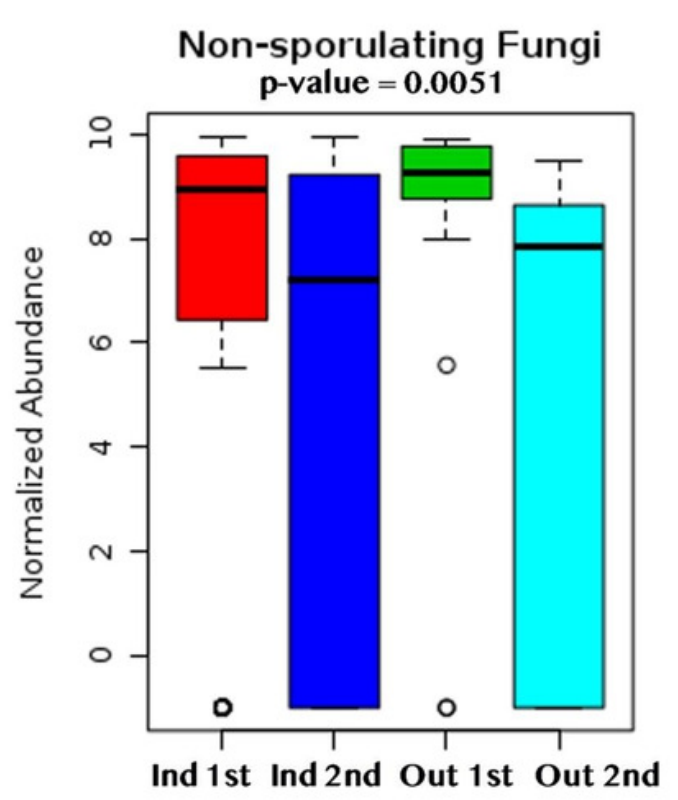

